\title{
Repensar y transformar las universidades desde su articulación y compromiso con las sociedades de las que forman parte ${ }^{1}$
}

\author{
Daniel Mato \\ Universidad Nacional de Tres de Febrero - \\ Consejo Nacional de Investigaciones \\ Científicas y Técnicas (CONICET). \\ dmato@untref.edu.ar
}

RECEPCIÓN: 03/10/18

ACEPTACIÓN FINAL: 05/11/18

\section{Resumen}

Este artículo expone resultados de una investigación realizada con la colaboración de los responsables de más de 200 experiencias de extensión universitaria y otras modalidades de vinculación social de equipos de 39 universidades públicas argentinas con comunidades y organizaciones sociales. Esta investigación llevó a determinar que la participación en la resolución de necesidades sociales contribuye a que las universidades mejoren tanto sus labores de generación de conocimientos como las de la formación profesional que ofrecen. Con base en esta premisa y en los aprendizajes derivados de sucesivos proyectos de investigación y procesos de acompañamiento desarrollados en colaboración con colegas de universidades interculturales e indígenas, he concluido que es necesario repensar y transformar todas las universidades desde su articulación y compromiso con las sociedades de las que forman parte.

Palabras clave: extensión universitaria compromiso social universitario, vinculación social universitaria, transformaciones universitarias, diversidad cultural
Rethinking and transforming universities on the basis of their connection with and commitment to their societies

\section{Abstract}

This article presents the results of a research project carried out with the collaboration of those responsible for more than 200 experiences of University Outreach and othe modalities of social partnership between teams from 39 Argentine public universities and communities and social organizations. This research project led to the conclusion that participation in the resolution of social needs contributes to the improvement of both the knowledge generation and the professiona training offered by universities. Based on this and the learning derived from successive research projects and accompanying processes developed in collaboration with colleagues from intercultural and indigenous universities, I have concluded that it is necessary to rethink and transform all universities from their connection with and commitment to the societies these institutions are part of.

Keywords: university extension, university social commitment, university social partnership, university transformations, cultural diversity.
Investigación y extensión universitaria / Perspectivas
Repensar e transformar as universidades desde sua articulação e compromisso com as sociedades das quais formam parte

\section{Resumo}

Este artigo expõe resultados de uma pesquisa realizada com a colaboração dos responsáveis de mais de 200 experiências de Extensão Universitária e outras modalidades de vinculação social de equipes de 39 universidades públicas argentinas com comunidades e organizações sociais. Esta pesquisa leva a concluir que a participação na resolução de necessidades sociais contribui para que as universidades melhorem tanto seu trabalho de produção de conhecimentos, como as da formação profissional que oferecem. Com base nisto e nas aprendizagens derivadas de sucessivos projetos de pesquisa e processos de acompanhamento desenvolvidos em colaboração com colegas de universidades interculturais e indígenas, concluí que é necessário repensar e transformar todas as universidades a partir de sua articulação e compromisso com as sociedades das quais formam parte.

Palavras-chave: extensão universitária, compromisso social universitário, vinculação social universitária, transformações universitárias, diversidade cultural.

Para citación de este artículo: Mato, D. (2018). Repensar y transformar las universidades desde su articulación y compromiso con las sociedades de las que forman parte. +E: Revista de Extensión Universitaria, 8(9), julio-diciembre, 38-52. doi: 10.14409/extension.v8i9.Jul-Dic.7837.

1) Este artículo presenta una versión ampliada de la conferencia de apertura ofrecida en el $8^{\circ}$ Congreso Nacional de Extensión Universitaria, realizado en la Universidad Nacional de San Juan, del 5 al 7 de septiembre de 2018. Deseo agradecer a la editora y pares evaluadores de la Revista $+\mathrm{E}$ por sus valiosos comentarios y sugerencias que han contribuido a mejorar la argumentación expuesta en estas páginas. 


\section{Introducción}

En Argentina, como en otros países latinoamericanos, las normativas vigentes establecen que las universidades tienen tres funciones sustantivas: docencia, investigación y extensión —u otras denominaciones análogas, según el país. No obstante, las visiones hegemónicas de la institucionalidad universitaria y las políticas públicas y prácticas institucionales derivadas de estas han posicionado a las labores de docencia e investigación en una suerte de pedestal y a las de extensión en un lugar subalterno. En este escenario, en los últimos años se ha venido planteando la necesidad de reconocer la importancia de las tres funciones institucionales por igual y, junto con ello, se han propuesto diversas estrategias orientadas al reconocimiento del valor de la extensión universitaria y la relevancia del compromiso social de las universidades.

En el contexto de estas iniciativas y debates asociados, he llevado adelante una investigación que produjo datos sobre más de 200 experiencias de vinculación de equipos de 39 universidades públicas argentinas con comunidades y organizaciones sociales. Esta pesquisa llevó a determinar que la participación en la resolución de necesidades sociales contribuye a que las universidades mejoren tanto su labor de generación de conocimientos como las de la formación profesional que ofrecen. Con base en estas conclusiones y en los aprendizajes derivados de sucesivos proyectos de investigación y procesos de acompañamiento que desde 2004 he desarrollado, en colaboración con colegas de universidades interculturales e indígenas en varios países latinoamericanos, he concluido también que es necesario repensar y transformar todas las universidades desde su articulación y compromiso con las sociedades de las que forman parte. Conviene adelantar que este tipo de modelo universitario fuertemente articulado y comprometido con la sociedad en la que está inserto es propio de las mencionadas universidades indígenas e interculturales. Afortunadamente, en años recientes, esta visión institucional ha venido inspirando el modelo adoptado por algunas universidades "convencionales", ${ }^{2}$ como también la Ley de Educación Superior de al menos un país latinoamericano, Ecuador. ${ }^{3}$ Es decir, cabe ser optimistas al respecto.

A efectos de que los resultados de la investigación realizada y argumentos derivados de la misma puedan ser interpretados apropiadamente, parece conveniente dejar constancia de que - desde un punto de vista institucional- he desarrollado mi trayectoria académica principalmente como investigador, frente a lo cual en apariencia mis labores de docencia y extensión habrían ocupado un lugar secundario. ${ }^{4}$ Pero en realidad esto no ha sido así, en especial en lo atinente al trabajo que suele denominarse de extensión, que personalmente prefiero llamar de vinculación social, o bien de articulación social o compromiso universitario. Desde los comienzos de mi labor he procurado trabajar en colaboración dialógica con,

2) Para simplificar la exposición, en este artículo denomino "convencionales" a las universidades que no han sido creadas explícitamente como indígenas o interculturales. Desde luego, esta manera tan genérica de denominarlas no da cuenta de las diferencias existentes entre muchas de ellas.

3) Ley Orgánica Reformatoria a Ley Orgánica de Educación Superior, disponible en: https://www.registroficial.gob.ec/ index.php/registro-oficial-web/publicaciones/suplementos/item/10802-suplemento-no-297.html

4) Desde 2010 soy Investigador Principal del Consejo Nacional de Investigaciones Científicas y Técnicas (CONICET), de Argentina. Entre 1990 y 2010 formé parte del Programa de Promoción del Investigador, del entonces denominado Consejo Nacional de Investigaciones Científicas y Tecnológicas (CONICIT), de Venezuela, en cuyo marco alcancé el rango superior del escalafón en el año 2000. 
y aprender de, los diversos grupos sociales con los que me he vinculado. ${ }^{5}$ Esta orientación de trabajo ha conducido a que desde hace ya varias décadas venga cuestionando el modelo hegemónico de universidad y de prácticas académicas. Pero, la tarea que desde 2004 he venido desarrollando en el campo de educación superior y pueblos indígenas y afrodescendientes $^{6}$ ha sido la experiencia que acabó por permitirme comprender más cabalmente que cuestionar el modelo de universidad hegemónico y tratar de trabajar en los intersticios que este ofrece no es suficiente, que es necesario transformar las universidades a partir de su articulación con los sectores sociales a los que pretende servir.

Esta conclusión provino justamente del acompañamiento cercano de procesos de creación y gestión de universidades por parte de organizaciones y/o de referentes de pueblos indígenas y afrodescendientes. Pero, aun antes de llegar a esa conclusión, inspirado por lo que venía observando en el desarrollo de esos tipos de experiencias, me formulé dos preguntas de investigación: ¿contribuyen las labores de extensión en sentido amplio (abarcador de voluntariado, investigación-acción, aprendizaje servicio, u otras modalidades de vinculación) al mejoramiento de las de docencia e investigación en las universidades "convencionales"? ¿Cómo lo hacen?

Estas dos preguntas fueron la base del proyecto de investigación con el que en 2010 regresé a Argentina y comencé mi trabajo de investigación desde el CONICET. Los resultados de esa investigación constituyen la base de lo expuesto en este artículo. Resulta significativo que la clave para el diseño de esta investigación provenga de mi experiencia de vinculación con actores sociales de fuera de los sistemas convencionales de educación superior. En este texto no expondré sobre esos otros tipos de experiencias de educación superior sobre los que he publicado múltiples artículos y coordinado nueve volúmenes colectivos, que cuentan con más de un centenar de capítulos a cargo de protagonistas destacados de esas experiencias, incluyendo numerosos profesionales indígenas y afrodescendientes. ${ }^{7}$ En cambio, me concentraré en presentar algunos resultados de dos proyectos de investigación específicamente dedicados a responder a las dos preguntas antes mencionadas.

5) La conferencia que ofrecí al recibir el Martin Diskin Memorial Lectureship Award, otorgado por OXFAM-America y la Latin American Studies Association (LASA), que sugestivamente titulé "Del 'diálogo de saberes' o a la construcción de modalidades de 'colaboración intercultural': Aprendizajes y articulaciones más allá de la Academia”, publicada posteriormente en el boletín de esta última asociación, ofrece reflexiones específicas respecto del vínculo entre mi trabajo de investigación y su articulación con mis labores junto a diversos actores sociales, para varias etapas de esta trayectoria (Mato 2017).

6) Comencé a trabajar específicamente en este campo en 2004 y en 2007 fui invitado por el Instituto Internacional de la Unesco para la Educación Superior en América Latina y el Caribe (UNESCO-IESALC, ver: http://www.iesalc. unesco.org.ve/index.php?option=com_content\&view=article\&id=22\&ltemid=405\&lang=es) a iniciar el Proyecto Educación Superior, Diversidad Cultural e Interculturalidad en América Latina). Este marco institucional me ha permitido familiarizarme con el desarrollo de casi dos centenares de experiencias de Educación Superior por/para/con pueblos indígenas y afrodescendientes y construir relaciones de colaboración con numerosos colegas que juegan papeles protagónicos en este campo; el cual incluye los casos de universidades indígenas e interculturales, entre otros modelos institucionales. En 2012 establecimos el Programa Educación Superior y Pueblos Indígenas y Afrodescendientes en América Latina en la Universidad Nacional de Tres de Febrero (UNTREF), desde el cual hemos impulsado la creación de la Red Educación Superior y Pueblos Indígenas y Afrodescendientes en América Latina (Red ESIAL; ver: http://untref.edu.ar/sitios/ciea/red-esial/), la cual actualmente cuenta con la participación de más de 60 instituciones de 11 países latinoamericanos. Estos procesos y el acompañamiento de numerosas experiencias de este campo constituyen las fuentes de mis aprendizajes sobre el tema.

7) Todas las publicaciones referidas están disponibles en los sitios en Internet del proyecto del UNESCO-IESALC y de la Red ESIAL indicados en la nota al pie $n^{\circ} 6$. A modo de ejemplos, acá me limitaré a hacer referencia a solo seis volúmenes colectivos (Mato, 2008, 2009, 2012, 2016, 2017, 2018) y dos artículos (Mato, 2008, 2015a). 
Cabe adelantar que esas investigaciones me llevaron a concluir que la participación en la resolución de necesidades sociales contribuye a que las universidades mejoren tanto su labor de generación de conocimientos, como las de la formación profesional que ofrecen. Es con base en esta premisa que considero que resulta necesario repensar y transformar las universidades desde su articulación y compromiso con las sociedades de las que forman parte.

Esta propuesta seguramente habrá de resultar aceptable para la mayoría de los colegas que trabajan en extensión universitaria. No obstante, no es como solemos encarar el asunto. Solemos ser bastante más tímidos y plantear que la extensión universitaria es una de las funciones fundamentales de las universidades y que tiene tanto valor como la docencia y la investigación. Este planteo es válido. Las tres funciones son importantes y no tenemos por qué invertir los términos del error y pretender que la extensión es más importante. Pero pienso que, si con sustento en nuestra propia experiencia e investigaciones específicas, estamos convencidos de que la participación en la resolución de necesidades sociales contribuye a que las universidades mejoren su calidad académica, entonces tenemos no el derecho sino el deber institucional de decirlo con toda claridad y argumentar al respecto.

Afirmar esto no significa pretender que la extensión es más importante que la docencia o la investigación, sino simplemente reconocer que la articulación y compromiso con las sociedades de las que nuestras universidades forman parte nos proveen una brújula para evaluar qué estamos haciendo, cómo lo estamos haciendo y procurar hacerlo mejor. Es decir, nos brinda orientación para repensar y transformar nuestras universidades. Si de verdad hemos llegado a esta conclusión, como es mi caso, y probablemente el de buena parte de quienes lean este artículo, tenemos el deber institucional, ético, epistemológico, didáctico y político de presentar nuestros argumentos al respecto ante otros sectores universitarios y de hacerlo con la mayor claridad y paciencia. El mismo deber lo tenemos en cuanto a las instancias gubernamentales que establecen políticas, presupuestos y programas para las universidades. También lo tenemos para lograr que esta conclusión sea comprendida por las agencias encargadas de evaluación y acreditación de las universidades de cada país.

Ahora bien, con explicar estas conclusiones no será suficiente. Queda por ver cómo llevarlas a la práctica a escala institucional agregada y cómo lograr su expresión concreta y efectiva en las políticas institucionales y públicas. Esto es, queda por ver cómo llevarlas a la práctica más allá del desarrollo de proyectos y programas específicos e incluso más allá de poner en ejecución estrategias más abarcadoras, como las de curricularización de la extensión.

No tengo dudas de que llevar adelante proyectos específicos y curricularizar la extensión son pasos necesarios, pero pienso que tenemos el deber de ir más allá, de anunciarlo y de generar reflexiones e intercambios al respecto. Es preciso repensar el papel de las universidades en tanto actores sociales comprometidos con las sociedades en las que están insertas. Esto no solo supone un trabajo hacia afuera sino también hacia adentro, que impulsará y demandará cambios institucionales, y mediante la reflexión y deliberación acerca de cómo realizar esa transformación.

Para contribuir a estos objetivos, en las próximas secciones de este artículo expondré de manera abreviada algunos resultados de la mencionada investigación, que conducen a determinar que la participación en la resolución de necesidades sociales contribuye a que las universidades mejoren su calidad académica y generen conocimientos. 


\section{Contribuciones de las experiencias de vinculación social de las universidades al mejoramiento de la calidad académica y factores que limitan su desarrollo y valoración institucional}

A continuación comentaré de manera sintética algunos resultados de la investigación que realicé entre 2011 y 2013, en cuyo marco produje y analicé información sobre de más de 200 experiencias de vinculación de 39 universidades públicas argentinas con comunidades y organizaciones sociales. Por razones de espacio, omitiré valiosos detalles expuestos en publicaciones anteriores (Mato 2013a, 2013b, 2015b). La primera etapa de esa investigación se basó en respuestas a un cuestionario con preguntas abiertas y a ser respondidas sin límite de extensión por los responsables de los equipos. La segunda etapa se basó en entrevistas no estructuradas hechas a más de veinte de ellos y en observación participante en dos congresos de extensión universitaria con participación de representantes de universidades de Argentina, Brasil y Uruguay. Corresponde especificar que los equipos protagonistas de las experiencias objeto de esta investigación estaban constituidos por docentes y estudiantes de diversas disciplinas y actuaban en contextos sociales muy disímiles entre sí.

Antes de presentar los resultados, me parece de interés apuntar que, según los casos, las experiencias estudiadas eran conceptualizadas por sus protagonistas en términos de: a) "extensión universitaria" (expresión que en buena parte de los casos suele ser acompañada de consideraciones que expresan reconceptualizaciones de la misma; b) "investigación-acción" (a veces planteada como "participativa"); c) "aprendizaje servicio" (en ocasiones especificado como "solidario"); d) "voluntariado", y e) "responsabilidad social universitaria".

\section{Principales resultados de la investigación realizada}

1) La investigación permitió concluir que las experiencias de vinculación de equipos universitarios con comunidades y organizaciones sociales contribuyen a la formación profesional ofrecida por las universidades así como a mejorar la calidad y pertinencia de la investigación que realizan dichos equipos.

2) Fue posible advertir que, pese a sus importantes contribuciones a la docencia e investigación, las actividades de extensión y otras modalidades de vinculación social no suelen ser valoradas apropiadamente por las universidades.

3) Se observó la importancia de algunos factores comunicacionales - hacia afuera y hacia adentro de las universidades - que limitan su mejor desarrollo y apropiada valoración institucional.

4) Muchos de los testimonios recogidos durante la investigación señalan la existencia de dificultades asociadas a la prevalencia de ideas de "calidad académica" que promueven que las universidades se dediquen exclusiva o principalmente a investigación y docencia, entendidas de maneras sesgadas. Sintéticamente, esos testimonios señalan:

a) Que la formación de profesionales brindada por las universidades mayormente se ajusta al perfil que demanda el mercado de empleo, ignorando demandas y propuestas de diversos sectores sociales así como las posibilidades de emprendimientos individuales o colectivos.

b) Que la realización de investigaciones está orientada a producir artículos para ser publicados en revistas académicas "dictaminadas" por pares, de alto "impacto", que 
son las que de un modo $\mathrm{u}$ otro acaban determinando temas, formatos y orientaciones epistemológicas y teóricas.

Respecto de este último punto en particular, no está de más reiterar un par de consideraciones que seguramente resultarán conocidas a buena parte de quienes lean este artículo:

i) En el marco de esas ideas academicistas, el "impacto" se mide por la cantidad de citas y/o referencias a los artículos de revistas académicas que se registran en artículos de otros autores publicados también en ese tipo de revistas. Esta manera endorreferencial de "medir" el impacto se desentiende por completo de considerar si esas publicaciones realizan algún tipo de contribución a la solución de problemas sociales, o bien técnicos, de interés social, político o económico, para la sociedad en general.

ii) Estas ideas de "calidad" y "excelencia", desvinculadas y desvinculantes de las demandas y propuestas de diversos sectores sociales, se ven reforzadas por la creciente importancia de los rankings universitarios internacionales en la formación de opinión pública general, así como, y muy especialmente, en sectores universitarios y de formulación de políticas y presupuestos.

\section{Principales conclusiones concretas de la investigación realizada}

Los testimonios recogidos durante esta investigación llevaron a determinar que las experiencias desarrolladas por equipos universitarios con comunidades y organizaciones sociales han contribuido a:

1) Mejorar la formación de los estudiantes, por ejemplo, mediante el desarrollo de diversos tipos de destrezas prácticas, puesta en práctica de conocimientos adquiridos en aula, construcción de nuevos conocimientos, desarrollo de perspectivas críticas respecto de conocimientos teóricos, desarrollo de destrezas de investigación, entre otros.

2) Identificar aspectos de la "realidad" no previstos en los programas de formación y que sería conveniente incluir en los programas de las asignaturas y/o planes de estudio para formar profesionales capaces de actuar en contextos reales de forma más efectiva.

3) Comprender la complejidad de problemas que no pueden resolverse desde perspectivas unidisciplinares y valorar la utilidad del trabajo interdisciplinario.

4) Desarrollar capacidades concretas para el trabajo interdisciplinario.

5) Adquirir nuevos conocimientos y destrezas útiles para el ejercicio profesional y/o el desarrollo de labores docentes.

6) Avanzar en proyectos de investigación existentes.

7) Identificar nuevas necesidades e intereses que habiliten nuevos proyectos de investigación.

8) Reconocer y valorar conocimientos no académicos que permiten enriquecer la formación profesional.

9) Identificar la existencia entre la población de algunos prejuicios y creencias que afectan la práctica profesional propia de las disciplinas de los miembros de los equipos universitarios participantes. 


\section{Fragmentos de uno de los testimonios recogidos en la investigación}

Para ilustrar, al menos parcialmente, los resultados expuestos, a continuación citaré textualmente fragmentos de las respuestas ofrecidas por la responsable de uno de los proyectos analizados. ${ }^{8}$ Se trata de una pregunta del cuestionario respecto de si estas experiencias habían facilitado la identificación de necesidades de formación o investigación y/o el desarrollo de aprendizajes en los equipos universitarios participantes:

"Nuestro grupo de trabajo viene desarrollando diversos proyectos que integran activamente a los estudiantes universitarios que manifiestan un gran entusiasmo. La participación de estudiantes voluntarios ha aumentado año a año, vemos un gran interés en sumarse a proyectos que demandan un compromiso con la comunidad. (...) La mayoría de ellos han manifestado que su participación en trabajos con la comunidad les permitió tanto el reconocimiento "en terreno" de las condiciones culturales y sanitarias de los pobladores de bajos recursos económicos como corroborar 'en la práctica' lo que leen en los libros de texto que utilizan para estudiar Medicina. La experiencia comunitaria les permitió tomar conciencia del compromiso social que tienen como futuros profesionales de la salud. En varias ocasiones han manifestado la necesidad de incorporar este tipo de actividades en el plan de la carrera de Medicina, entienden que es necesario que la Universidad se acerque a la comunidad a través de este tipo de actividades estableciendo puentes de comunicación que benefician tanto a docentes y estudiantes como a la propia comunidad a través de un trabajo organizado y enmarcado formalmente. Ellos mismos comentan que no son "los mismos" antes y después de haber participado en el proyecto. (...) Para los estudiantes universitarios significa un entrenamiento donde lo humano es prioritario. Entender al otro como un par, humanizar el trato con el otro, escucharlo y entenderlo. Para los docentes, además, fue también una práctica interactuar con las autoridades de la Unidad Sanitaria y las escuelas. Conocer sus estructuras, su dinámica y prioridades, comprender sus tiempos. El desarrollo de este proyecto nos demandó un período de adaptación a las otras instituciones participantes que en este caso están más atentas a necesidades concretas del día a día en la problemática sanitaria y educativa de la gente del barrio que a generar a futuro un cambio beneficioso para su gente. La realidad los desborda y el proyecto tuvo que ajustarse a la situación local sin dejar que se desdibuje su objetivo central". (Entrevista a Betina Cecilia Pezzani, 20/09/2012)

\section{Acerca de las diferencias entre la visión institucional de diversos actores universitarios}

En publicaciones anteriores he explicado con ejemplos concretos que las organizaciones complejas no son homogéneas sino heterogéneas, en el sentido de que en su interior existen diferentes interpretaciones del sentido, visión, misión y valores de las mismas. Así, cabe decir que al interior de organizaciones complejas conviven y contienden diversas culturas institucionales (Mato, Maldonado y Rey, 2011; Mato, 2012).

Las universidades no son una excepción a esta suerte de regla. Dentro de ella existen diversas interpretaciones acerca de su función social, o de "la ciencia", de las relaciones

8) Al enviar sus respuestas, la colega en cuestión, como la casi totalidad de los responsables de proyectos, autorizó que mencionara su nombre en las publicaciones resultantes. En este caso, se trata de la Dra. Betina Cecilia Pezzani, de la cátedra de Microbiología y Parasitología de la Universidad Nacional de La Plata. 
ciencia-otros saberes, relaciones conocimiento teórico y aplicado, relaciones docencia-investigación-extensión u otras modalidades de vinculación con la comunidad. Significativamente, un buen número de respuestas al cuestionario de nuestro proyecto revela no solo la presencia al interior de las universidades de interpretaciones diversas sobre esos asuntos, sino también la preeminencia en ellas de visiones academicistas que no valoran de manera apropiada las actividades desarrolladas por los proyectos de extensión, voluntariado, u otras formas de vinculación social universitaria.

Para ilustrar estas circunstancias, vuelvo a citar fragmentos de otra respuesta de la ya mencionada Dra. Betina Cecilia Pezzani, quien sostuvo:

"Muchas veces hemos sido subestimadas como docentes investigadoras por docentes superiores y pares de nuestro lugar de trabajo por presentarnos en las distintas convocatorias (se refiere a convocatorias para proyectos de extensión y de voluntariado, D.M.) percibimos ese dejo de no estar haciendo algo valioso como escribir un paper o ir a un Congreso, que se perdía el tiempo. De hecho, se nos ha preguntado personalmente si queríamos dejar de hacer investigación. Muy por el contrario, nuestra producción científica no mermó y hemos podido organizar todas las tareas sin desmedro de nuestras funciones como docentes con dedicación exclusiva. Nada nos frenó, muy por el contrario, nos reforzó la idea de que la universidad tiene que estar con todos sus recursos humanos, docentes y estudiantes, cerca de la gente, escuchando, conteniendo, asistiendo y aportando soluciones a problemas concretos que en la mayoría de los casos surgen de la propia comunidad. Nuestro proyecto articuló armónicamente la extensión con la docencia y la investigación. Seguiremos participando en todo proyecto que contemple nuestro compromiso con la comunidad".

No podemos citar aquí otras respuestas a los cuestionarios pero, para generalizar, puede afirmarse que gran parte de ellas permite observar la existencia al interior de las universidades de diferentes interpretaciones respecto de su papel en la sociedad y cómo cumplirlo. ${ }^{9}$ Esas respuestas también dan cuenta del hecho de que las visiones predominantes en la mayoría de las universidades de referencia no son precisamente las que valoran el tipo de proyectos de trabajo con la comunidad y que hay obstáculos para desarrollar proyectos con grupos de población y sus organizaciones.

La investigación realizada lleva a concluir que las visiones cientificistas continúan siendo hegemónicas y que los obstáculos al desarrollo de actividades con grupos de población persisten, incluso pese a que en años recientes en Argentina han ocurrido interesantes avances en la valoración institucional de las labores de extensión universitaria y vinculación social.

\section{Obstáculos a la vinculación social y colaboración intercultural en educación superior Muchas universidades argentinas y de otros países latinoamericanos han venido avanzando en pos de otorgar mayor valoración a las labores de extensión y de repensar la idea de extensión universitaria. Sin embargo, las respuestas a los cuestionarios de la investigación}

9) En mis publicaciones anteriores sobre el tema pueden encontrarse fragmentos de las respuestas ofrecidas por varias decenas de colegas (Mato, 2013a, 2013b, 2015b). 
realizada en Argentina llevan a determinar que en la mayoría de los casos estos movimientos aún no resultan suficientes para cambiar de modo significativo un modelo de universidad que lleva siglos hegemónicamente instalado como parte de la cultura institucional.

Se trata de un modelo de universidad academicista cuya escasa valoración por la vinculación social, en las últimas décadas, se ha visto fortalecida en especial por dos tipos de factores. Por un lado, por ciertas políticas de estímulo a la investigación que evalúan logros preeminentemente mediante publicaciones, citas y patentes. Por otro lado, por la creciente ascendencia de los rankings internacionales de universidades en la opinión pública en general y en particular en algunos sectores universitarios, así como en los organismos de formulación de políticas universitarias y en los de toma de decisiones presupuestarias.

En contraste con la preeminencia de ese modelo academicista y la importancia de los mencionados factores, la investigación realizada evidencia que las experiencias de colaboración entre equipos universitarios y comunidades y organizaciones sociales no solo contribuyen a mejorar la calidad de vida de sectores sociales sino también la formación profesional ofrecida por las universidades, así como las posibilidades y calidad de investigación que realizan dichos equipos. No obstante, estos tipos de actividades no suelen contar con valoración institucional.

Los problemas propios de este modelo academicista no son nuevos. Se asientan en procesos de larga data asociados a la hegemonía de ciertas ideas de universidad y de ciencia.

No se trata simplemente de una coyuntural orientación de políticas que suelen calificarse como neoliberales, como si la sola aplicación de ese adjetivo fuera suficientemente explicativa. Hay que ser cautos con estas representaciones simplistas del problema, porque esa rápida adjetivación obstaculiza las posibilidades de profundizar el análisis. Los problemas que nos preocupan tienen una historia larga y menos fácil de cambiar. Provienen de "una manera de ver el mundo", de ciertas formas de "sentido común", son parte de una determinada episteme. En los contextos institucionales en los cuales se producen y reproducen representaciones y políticas de educación superior, y de ciencia y tecnología (incluyendo agencias gubernamentales dedicadas a estos temas, universidades e institutos nacionales de investigación), las referencias explícitas o implícitas a la existencia de dos clases de saber, uno solo de los cuales tendría validez universal, mientras que el otro (diverso a su interior) no la tendría, resultan hegemónicas, y esto ha sido así desde sus orígenes.

Esas representaciones que expresan una visión de mundo se han convertido en "sentido común", y es a partir de ellas que se formula la mayoría de las políticas y normas que regulan los campos de educación superior y ciencia y tecnología. Más aún, frecuentemente este sistema de representaciones incluye una cierta visión de la ciencia según la cual existen unas "ciencias duras" y otras a las que, por contraposición, no quedaría más alternativa que llamar "blandas".

Pensar la producción y validez de conocimiento divididas en dos mundos, uno solo de los cuales sería poseedor de verdades "universales" y el otro de verdades que, en contraposición con la postulada y pretendida universalidad de aquellas, solo cabría asumir como "Iocales", es tan antigua como el credo en la superioridad del "hombre blanco" y la "civilización occidental", que pretendidamente sería la generadora y poseedora de tal saber "universal".

Los encuentros, conflictos y negociaciones entre ese saber "universal" y esos otros que se representan como si fueran meramente "locales", se han hecho más frecuentes en las úl- 
timas décadas por la creciente extensión y profundización de relaciones entre actores sociales que desarrollan sus prácticas a escala mundial, o en algunos casos continental o regional supranacional, y los que las desarrollan a escalas más "locales", sean estas nacionales, provinciales, municipales o comunitarias. El análisis de las limitaciones y consecuencias de la creencia en la existencia de un saber pretensiosa y pretendidamente "universal" y otros de validez apenas "local" resulta no solo más necesario sino también más factible en el marco de los procesos de globalización contemporáneos debido a la creciente importancia de los intercambios entre actores sociales cuyas maneras de ver del mundo, producir conocimiento y accionar, se forman en muy diversos contextos y dan lugar a distintos tipos de saber. Por ello, la colaboración intercultural tanto en la producción de conocimientos como en la formación de profesionales y técnicos se hace cada día más necesaria y también más factible.

Sin embargo, a pesar de los ya no tan recientes análisis y argumentaciones acerca los condicionantes históricos y contextuales del conocimiento científico, podemos observar que las prácticas y discursos de numerosos investigadores, instituciones de investigación y formación académica y organismos de formulación de políticas de educación superior, ciencia y tecnología, se asientan, cuanto menos implícitamente, en la idea de que "la ciencia", como modo de producción de conocimientos, y el "conocimiento científico" como acumulación de conocimientos producidos "científicamente", tendrían validez "universal". Es decir, según las instituciones e investigadores alineados con esa visión de mundo, esos conocimientos resultarían verdaderos y aplicables en cualquier tiempo y lugar.

En el marco de esa visión de mundo, la otra clase abarcaría una amplia diversidad de tipos de saber, de modos de producción de conocimiento y sus resultados, a los cuales, en contraposición con el saber "universal" de la "ciencia", suele caracterizarse, según los casos, como "étnicos" o "locales", en cualquier caso como saberes "particulares", "no-universales". Así, estos "otros saberes" solo tendrían validez y aplicación "local" al menos hasta tanto sean validados con los métodos propios de "la ciencia".

Un ejemplo significativo de los problemas que esto plantea es la evaluación y validación de conocimientos "étnicos" y otros calificados simplemente como "locales" sobre las aplicaciones terapéuticas de especies vegetales mediante métodos "científicos", a lo que, de manera significativa, suele seguir su apropiación y patentado por instituciones "científicas" y, con más frecuencia, por corporaciones farmacéuticas (Blum, 1993; Brush, 1993; Greene, 2004; Nigh, 2002).

Semejante efecto práctico de esta división no puede pasarse por alto, como tampoco puede pasarse por alto que en las universidades la investigación y docencia sobre, por ejemplo, medicina e instituciones políticas, jurídicas y económicas, propias de esos "otros" grupos humanos, suelan quedar reservadas a los departamentos y escuelas de Antropología y solo de modo excepcional sean materia de investigación y formación en departamentos y escuelas de Derecho, Ciencias Políticas, Economía, Medicina, o de las que vengan al caso según el tipo de saber en cuestión.

En los últimos años han surgido voces que postulan que, con el propósito de construir sociedades más "incluyentes", las universidades deberían ofrecer formación en esos "otros saberes", pero por lo general esto se ve en términos de concesión a esos "otros" grupos humanos. Esta visión condescendiente pierde de vista que la colaboración intercultural resulta imprescindible tanto para quienes formamos parte de las instituciones que producen 
conocimiento cuyo valor supuestamente tendría carácter "universal" (las universidades y otros centros de producción de conocimiento "científico") como para quienes desarrollan sus prácticas en otros tipos de marcos institucionales y sociales y producen conocimientos que suelen calificarse como "locales" o "particulares".

Pensar la idea de colaboración intercultural en educación superior y en ciencia y tecnología y, en conexión con esto, en la incorporación de otros saberes y formas de producción de conocimientos, como una condescendiente política de "inclusión", resulta pobre y autoempobrecedoramente racista. No se trata de hacer concesiones sino de reconocer las limitaciones de esas visiones racistas para poder superar el monoculturalismo y desarrollar perspectivas de investigación y de formación de profesionales y técnicos que sean pertinentes con la diversidad cultural que caracteriza a las sociedades contemporáneas (Mato, 2008; 2009).

El problema que se confronta no es simplemente de políticas universitarias, es más profundo. Se relaciona con la hegemonía de valores que operan como creencias entre no pocos científicos "serios", docentes universitarios y autoridades, y tomadores de decisiones en este campo. Un ejemplo saliente lo constituyen las pretensiones de objetividad del conocimiento científico y la neutralidad de valores de los investigadores. La aplicación de estas ideas, provenientes de las llamadas "ciencias duras", a las llamadas "ciencias sociales" conduce a ignorar cómo nuestra subjetividad es constitutiva de nuestro trabajo de investigación. La pretensión de objetividad supone ante todo cerrar los ojos a algunas formas ineludibles de subjetividad, como, por ejemplo, la que necesariamente afecta al planteamiento de un problema, la formulación de las preguntas que han de guiar la investigación, el desarrollo de una perspectiva de análisis y el establecimiento de relaciones con las personas cuyas prácticas estudiamos. Estos aspectos que la labor investigación plantea no pueden ser resueltos de manera "objetiva", al menos no en las pesquisas que se desarrollan desde las llamadas humanidades y ciencias sociales. Los modos de responder a los retos que cada una de esas tareas del trabajo de investigación establece dependerán del lugar desde el cual se investigue, de para qué se investigue y de lo que se pretenda hacer con los resultados de la investigación. Pero estas preguntas no siempre se plantean. Antes bien, el trabajo de investigación académica suele desarrollarse de forma compulsiva respecto de ellas, es decir, sin reflexión. Las respuestas están allí instaladas con la firmeza y "naturalidad" de un credo, de modo tal que este tipo de preguntas no se formulan sino excepcionalmente.

El problema es que quienes impulsan las concepciones cientificistas parecen no comprender que tanto las propias preguntas de investigación como los modos de producción de datos dependen en última instancia de opciones epistemológicas, las cuales están asociadas a visiones de mundo y posiciones éticas y políticas que, a su vez, dependen, entre otros factores, del tipo de relaciones que se sostiene o se aspira a sostener con actores sociales extraacadémicos. Las posiciones éticas y políticas son constitutivas del basamento epistemológico y de las perspectivas teóricas de nuestras investigaciones, y así también de las preguntas y de los métodos.

Las preguntas de investigación no son las mismas, ni tampoco los métodos, si lo que se pretende es "escribir" estudios supuestamente "objetivos" o si se quiere producir algún tipo de saber útil a los intereses de actores sociales extraacadémicos. De las respuestas a preguntas del tipo ¿para qué y para quiénes investigar? depende qué investigar, cómo, con quiénes, en el marco de cuáles relaciones, con cuáles propósitos. 
El caso es, entonces, que el cientificismo acarrea dos tipos de problemas a las universidades. Por un lado, obstaculiza que otros modos de conocimiento y las prácticas profesionales extraacadémicas puedan ser objeto de mejor valoración y cuidadosa articulación y aprovechamiento en la investigación y formación universitarias. Por otro lado, acaba por afectar la pertinencia y legitimidad social de la formación y prácticas universitarias y por excluir oportunidades de intercambio, aprendizaje y participación en algunas dinámicas sociales.

En contraste con estos problemas propios de las orientaciones de trabajo cientificistas, las modalidades de colaboración entre equipos universitarios y diversos sectores de población pueden contribuir a mejorar la calidad de vida de la población así como la formación de profesionales y el desarrollo de investigación y aplicaciones. Las más de doscientas experiencias que sirven de referencia a la investigación que funda a este artículo constituyen ejemplos de ello.

Así, cabe reiterar que la investigación realizada lleva a concluir que, no solo por razones sociales, sino también por razones académicas, para mejorar tanto la calidad de la formación profesional que ofrecen como la calidad de la investigación que realizan, parece necesario repensar y transformar las universidades (el modelo universitario mismo) desde su articulación y compromiso con las sociedades de las que forman parte.

El análisis de los procesos en curso en este campo hace plausible pensar que esta propuesta transformadora es viable, ya que en varios países latinoamericanos se viene avanzando de maneras cónsonas con lo planteado en este artículo. En este sentido, a modo de ejemplo, resulta destacable el valor que la recientemente promulgada Ley Orgánica Reformatoria a Ley Orgánica de Educación Superior de la República de Ecuador ${ }^{10}$ otorga a la labor de vinculación social de las universidades y otras instituciones de educación superior, y el peso que esta adquiere respecto del concepto de calidad y su aseguramiento y evaluación por parte del organismo pertinente. De maneras diversas pero convergentes, en países como Argentina, Uruguay y Brasil, ha venido desarrollándose un proactivo movimiento de valorización de la extensión y han crecido las propuestas y experiencias de curricularización de la misma (Cano, 2016, Carneiro et al., 2014, Maciel, 2010, Schmidt, 2009, Tomassino et al., 2010).

En Argentina, especialmente en la última década, ha habido avances importantes, entre los que resalta el incremento de los presupuestos destinados a extensión universitaria, la creación de la Red Nacional de Extensión Universitaria (REXUNI) en el marco del Consejo Interuniversitario Nacional (CIN), y la realización hasta la fecha de ocho congresos nacionales de extensión universitaria, en cada uno de los cuales se han presentado centenares de ponencias que muestran esos avances en el campo en numerosas universidades nacionales y de otros países latinoamericanos. A efectos de este artículo, entre estos avances viene al caso destacar los registrados en cuanto a la curricularización de la extensión y de las prácticas sociocomunitarias, así como al lugar institucional asignado a la extensión, a las prácticas sociocomunitarias, al aprendizaje-servicio y otras modalidades de vinculación social en algunas universidades (Cecchi, Pérez y Sanllorenti, 2013; Denazis, 2016; Herrera, 2013;

10) La Ley Orgánica Reformatoria a Ley Orgánica de Educación Superior de la República de Ecuador (promulgada el 2 de agosto de 2018) está disponible en: https://www.registroficial.gob.ec/index.php/registro-oficial-web/publicaciones/suplementos/item/10802-suplemento-no-297.html 
Martínez, 2008; Ramírez y Gantus, 2013; UNL, 2018), ${ }^{11}$ como también a la creciente elaboración conceptual al respecto. ${ }^{12}$ Todo ello se ha visto acompañado por la incorporación, por parte de la Comisión Nacional de Evaluación y Acreditación Universitaria (CONEAU), de las actividades de extensión como una de las dimensiones de sus protocolos de evaluación y criterios de acreditación.

Adicionalmente, es necesario considerar que estos avances desde y/o con relación a la extensión se han dado de manera convergente con otros que desde hace ya varias décadas se observan en el ámbito de las prácticas de investigación, acerca de lo cual suele reconocerse la creciente incidencia de las contribuciones pioneras de Orlando Fals Borda (1979; 1986) al desarrollo de las perspectivas de Investigación-Acción-Participativa. Del mismo modo, han crecido las interpelaciones que diversos actores y movimientos sociales dirigen a las universidades reclamando que asuman papeles de mayor compromiso con las necesidades, demandas y propuestas de las sociedades de las que forman parte.

\section{Algunas preguntas para estimular reflexiones sobre lo expuesto}

Pienso que la mejor forma de concluir este artículo es proponiendo algunas preguntas que sirvan para estimular reflexiones acerca de cómo transformar nuestras universidades a partir de profundizar su articulación y compromiso con las sociedades en las que están insertas:

¿Cómo podrían las propuestas transformadoras expresarse en la formulación de la misión social de cada universidad?

¿Qué formas concretas podrían tomar en las instancias de deliberación y gobierno universitario?

¿Sería posible hacerlo a través de consejos sociales?

¿Cómo hacer lugar apropiadamente a la diversidad de orientaciones de trabajo desde las cuales se plantean y atienden distintas modalidades de extensión universitaria y, en general, de compromiso y vinculación con la sociedad?

¿Cómo manejar las relaciones entre diferentes formas de producción de conocimientos?

¿Cómo construir relaciones de colaboración con otros actores sociales (y, en este sentido, con frecuencia interculturales) que resulten mutuamente provechosas, fértiles y duraderas?

¿Existen acaso respuestas genéricas aplicables por igual a todas las universidades en todos los contextos sociales?

¿Cómo perfilar políticas públicas que reconozcan y valoren las diferencias entre diversos contextos y modelos universitarios?

¿Cómo lograr que estas diferencias se reconozcan y valoren en los criterios de evaluación y acreditación de universidades y carreras?

¿Cómo hacerlo en las políticas de aseguramiento de la calidad?

11) Respecto de estos tipos de avances cabe mencionar los puestos en práctica por ejemplo en la Universidad Nacional de Avellaneda, la Universidad Nacional de Entre Ríos (UNER), la Universidad Nacional del Litoral (UNL) y la Universidad Nacional de Mar del Plata (UNMdP), entre otras.

12) Desde 2011, la Revista $+E$, a cuya consideración se ha sometido este artículo, ha venido construyendo y difundiendo una extensa y valiosa colección de artículos que permite observar estos avances. Además, ella misma juega un importante papel en este proceso y constituye a la vez un significativo indicador del creciente desarrollo de este campo en América Latina. 


\section{Referencias bibliográficas}

Blum, E. (1993). Making biodiversity conservation profitable. A case study of the Merck/INBio Agreement. Environment, 35(4), 16-45.

Brush, S.B. (1993). Indigenous Knowledge of Biological Resources and Intellectual Property Rights: The Role of Anthropology. American Anthropologist, 95(3), 653-671.

Cano, A. (2016). Debates de ayer y hoy: algunos antecedentes de las nociones de extensión e integralidad en la Universidad de la República. InterCambios, 3(1), 13-23.

Carneiro, P.; Collado D. y Oliveira, N. (2014). Interfaces - Revista de Extensão, 2(3), 4-26. Universidade Federal de Minas Gerais.

Cecchi, N.; Pérez, D. y Sanllorenti, P. (2013). Compromiso Social Universitario. De la universidad posible a la universidad necesaria. Buenos Aires: Instituto de Estudios y Capacitación de I Federación Nacional de Docentes Universitarios.

Denazis, J. (2016). La gestión académica en la universidad: Integración de programas orientados a la calidad, inclusión, mejora continua, flexibilidad e innovación. En Gudiño, P. y Escala, M. (Comps.). Innovación Interamericana en Educación Superior (pp. 266-284). Santo Domingo, R. Dominicana: Organización Universitaria Interamericana

Fals Borda, O. (1979). El problema de cómo investigar la realidad para transformarla. Bogotá: Tercer Mundo.

(1986). Conocimiento y Poder Popular, Lecciones con campesinos de Nicaragua, México y Colombia. Bogotá: Siglo XXI Editores.

Greene, S. (2004). Indigenous People Incorporated? Culture as Politics, Culture as Property in Pharmaceutical Bioprospecting. Current Anthropology, 4(2), 211-224.

Herrera, M.L. (2013). Una mirada sobre la extensión universitaria en Argentina. Recuperado de http://www. bdigital.unal.edu.co/7472/1/marialilianaherreraalbrieu.20121.pdf

Maciel, L.R. (2010). Política Nacional de Extensão: perspectivas para a universidade brasileira. Revista Participação, 10(18), 15-25. Universidade de Brasília.

Martínez, M. (Ed.) (2008). Aprendizaje servicio y responsabilidad social de las universidades.

España: Ministerio de Educación y Ciencia y Ediciones Octaedro.

Mato, D. (2008). No hay saber "universal", la colaboración intercultural es imprescindible. Alteridades, 18(35), 101-116. Departamento de Antropología, Universidad Autónoma Metropolitana-Iztapalapa, México.

- (2012). Heterogeneidad social e institucional, interculturalidad y comunicación intercultural. MATRIZes, 6(1), 43-61. Programa de Postgrado en Estudios de la Comunicación, Universidad de Sao Paulo.

(2013a). Aprendizajes de equipos universitarios en experiencias de colaboración con comunidades y organizaciones sociales, realizadas con apoyo del Programa de Voluntariado Universitario de Argentina, en 2008. Apuntes. Revista de Ciencias Sociales, XL(72), 33-56. Universidad del Pacífico, Perú.

- (2013b). Contribución de experiencias de vinculación social de las universidades al mejoramiento de la calidad académica y factores que limitan su desarrollo y valoración institucional. Revista Avaliação: Revista da Avaliação da Educação Superior, 18(1), 151-180. Universidade de Sorocaba, Brasil.

(2015a). Pueblos Indígenas, Estados y Educación Superior. Aprendizajes de experiencias en varios países de América Latina potencialmente útiles a los procesos en marcha en Argentina. Cuadernos de Antropología Social, (41), 5-23. Facultad de Filosofía y Letras. Instituto de Ciencias Antropológicas. Universidad de Buenos Aires.

— (2015b). Vinculación social universitaria en Argentina. Diversidad de orientaciones de trabajo, logros y dificultades de las experiencias apoyadas por el Programa Nacional de Voluntariado Universitario. CPU-e, Revista de Investigación Educativa, 20, 132-149. Universidad Veracruzana, México. 
(2017). Del "diálogo de saberes" a la construcción de modalidades de "colaboración intercultural": Aprendizajes y articulaciones más allá de la Academia. LASA Forum, 48(3), 8-17.

(Coord.) (2008). Diversidad cultural e interculturalidad en Educación Superior. Experiencias en América Latina. Caracas: UNESCO-IESALC.

— (2009). Educación Superior, Colaboración Intercultural y Desarrollo Sostenible/Buen Vivir. Experiencias en América Latina. Caracas: UNESCO-IESALC.

(2012) Educación Superior y Pueblos Indígenas y Afrodescendientes en América Latina. Normas, Políticas y Prácticas Caracas: UNESCO-IESALC.

(2016) Educación Superior y Pueblos Indígenas en América Latina. Experiencias, interpelaciones y desafíos. Sáenz Peña (Provincia de Buenos Aires): EDUNTREF y México DF: Universidad Nacional Autónoma de México-UNAM.

(2017). Educación Superior y Pueblos Indígenas y Afrodescendientes en América Latina. Políticas y prácticas de inclusión, democratización, e interculturalización. Sáenz Peña, Buenos Aires: EDUNTREF y José María Morelos, Quintana Roo: Universidad Intercultural Maya de Quintana Roo.

(2018). Educación Superior, Diversidad Cultural e Interculturalidad en América Latina. Caracas: UNESCO-IESALC y Córdoba: Universidad Nacional de Córdoba.

Mato, D.; Maldonado, A. y Rey, E. (2011). Interculturalidad y Comunicación Intercultural. Propuesta teórica y estudio de experiencias de participación social en la gestión de servicios públicos en una comunidad popular de la ciudad de Caracas. Caracas: Universidad Central de Venezuela.

Nigh, R. (2002). Maya Medicine in the Biological Gaze Bioprospecting Research as Herbal Fetishism. Current Anthropology, 43(3), 451-464.

Ramírez, G. y Gantus, D. (2013). Jerarquización de la Extensión en la UNER. Apuntes para pensar la curricularización de la Extensión en contexto. Documento preliminar de discusión. Recuperado de http://beu.extension. unicen.edu.ar/xmlui/handle/123456789/129

República del Ecuador, Asamblea Nacional (2 de agosto de 2018). Ley Orgánica Reformatoria a Ley Orgánica de Educación Superior. Suplemento del Registro Oficial, 2(297).

Schmidt, J.P. (Org.) (2009). Instituições Comunitárias: Instituições públicas não-estatais. Santa Cruz do Sul: EDUNISC.

Secretaría de Extensión Universitaria, Universidad Nacional del Litoral (UNL) (2018). Informe de Gestión 20142018. Santa Fe: Universidad Nacional del Litoral. Recuperado de https://www.unl.edu.ar/extension/wpcontent/uploads/sites/4/2016/11/Extensi\%C3\%B3nInformeGesti\%C3\%B3n2014-2018web-1.pdf

Tommasino, H.; Cano, A.; Castro, D.; Santos, C. y Stevenazzi, F. (2010). De la extensión a las prácticas integrales. En Hacia la Reforma Universitaria. La extensión en la transformación de la enseñanza: Los Espacios de Formación Integral. Montevideo: Rectorado de la Universidad de la República. 